British Journal of Nutrition (2012), 108, 1764-1772

doi:10.1017/S0007114511007288

(C) The Authors 2012. The online version of this article is published within an Open Access environment subject to the conditions of the Creative Commons Attribution-NonCommercial-ShareAlike licence $<$ http://creativecommons.org/licenses/by-nc-sa/2.5/ $>$. The written permission of Cambridge University Press must be obtained for commercial re-use.

\title{
A high-fat diet containing whole walnuts (Juglans regia) reduces tumour size and growth along with plasma insulin-like growth factor 1 in the transgenic adenocarcinoma of the mouse prostate model
}

\author{
Paul A. Davis ${ }^{1 *}$, Vihas T. Vasu ${ }^{2} \dagger$, Kishorchandra Gohil ${ }^{2}$, Hyunsook Kim ${ }^{1}$, Imran H. Khan ${ }^{3}$, \\ Carroll E. Cross ${ }^{2}$ and Wallace Yokoyama ${ }^{4}$ \\ ${ }^{1}$ Department of Nutrition, University of California, Davis, CA 95616, USA \\ ${ }^{2}$ Department of Internal Medicine, Division of Pulmonary and Critical Care Medicine, University of California, Davis, \\ CA 95616, USA \\ ${ }^{3}$ Department of Pathology and Laboratory Medicine, Center for Comparative Medicine, University of California, Davis, \\ CA 95616, USA \\ ${ }^{4}$ Processed Foods Research, US Department of Agriculture, Western Regional Research Center, Agricultural Research Service, \\ Albany, CA 94710, USA \\ (Submitted 10 October 2011 - Final revision received 6 December 2011 - Accepted 6 December 2011 - First published online 16 January 2012)
}

\begin{abstract}
Prostate cancer (PCa) has been linked to fat intake, but the effects of both different dietary fat levels and types remain inconsistent and incompletely characterised. The effects on PCa in the transgenic adenocarcinoma of the mouse prostate (TRAMP) cancer model of an elevated fat ( $20 \%$ of energy as fat) diet containing $155 \mathrm{~g}$ of whole walnuts were compared to those of an elevated fat ( $20 \%$ of energy as soyabean oil) diet with matched macronutrients, tocopherols as well as a low-fat ( $8 \%$ of energy as soyabean oil) diet. Mice, starting at 8 weeks of age, consumed one of the three different diets ad libitum; and prostates, livers and blood were obtained after 9, 18 or 24 weeks of feeding. No differences were observed in whole animal growth rates in either high-fat (HF) diet group, but prostate tumour weight and growth rate were reduced in the walnut diet group. Walnut diet group prostate weight, plasma insulin-like growth factor 1, resistin and LDL were lower at 18 weeks, while no statistically significant prostate weight differences by diet were seen at 9 or 24 weeks. Multiple metabolites in the livers differed by diet at 9 and 18 weeks. The walnut diet's beneficial effects probably represent the effects of whole walnuts' multiple constituents and not via a specific fatty acid or tocopherols. Moreover, as the two HF diets had dissimilar effects on prostate tumour growth rate and size, and yet had the same total fat and tocopherol composition and content, this suggests that these are not strongly linked to PCa growth.
\end{abstract}

Key words: Prostate cancer: Transgenic adenocarcinoma of the mouse prostate model: Fat: Whole foods: Insulin-like growth factor 1: Chemoprevention

Prostate cancer (PCa), a leading cause of male cancer deaths $^{(1)}$, has been linked to fat intake ${ }^{(2)}$, but the effects of both dietary fat levels and differences in unsaturated fatty acids on prostate as well as other cancers remain inconsistent and poorly characterised. Studies have suggested that $n-3$ fatty acids inhibit ${ }^{(3-5)}$ or have no effect ${ }^{(6,7)}$ on PCa, while $n-6$ fatty acids stimulate PCa development ${ }^{(8)}$. Hori et $a l^{(9)}$, in a very recent review, concluded that there was little evidence to suggest that a high-fat (HF) diet is associated with increased PCa risk, but still suggested that reduced dietary fat intake in combination with a healthy diet and lifestyle may slow the progression of PCa in certain patients. Although high in fat, walnut fats are mainly polyunsaturated and the effects of consuming walnuts on PCa are unclear ${ }^{(10)}$. In addition, Freedland and co-workers have reported that mouse PCa xenograft tumour growth is not associated with dietary fat content ${ }^{(11,12)}$. Moreover, Hardman \& Ion ${ }^{(13)}$ have shown that implanted MDA-MB 231 human breast cancer growth in nude mice was significantly less in the group consuming walnuts (human equivalent of two servings a day) compared to a control group fed maize oil and have just reported that walnuts suppress mammary gland tumorigenesis in the

Abbreviations: GUI, genitourinary intact; HF, high-fat diet; IGF-1, insulin-like growth factor 1; LF, low-fat diet; NE, neuroendocrine; PCa, prostate cancer; TRAMP, transgenic adenocarcinoma of the mouse prostate; WW, whole walnut diet.

*Corresponding author: Dr P. A. Davis, fax +1 530752 8966, email padavis@ucdavis.edu

†Present address: Department of Medicine, Division of Oncology, National Jewish Health, Denver, CO 80 206 , USA. 
C(3)1 TAg mouse but the effect could not be accounted for by the $n-3$ content alone ${ }^{(13,14)}$.

The present study evaluated the effects of whole walnuts fed as part of a moderately HF diet on tumour growth in the transgenic adenocarcinoma of the mouse prostate (TRAMP) animal cancer model ${ }^{(15)}$. The high-fat, non-walnut diet was formulated using tocopherol-stripped soyabean oil to achieve not only the same fat levels but supply substantially similar fatty acid profiles as well. The experimental and control diets' $\alpha$ - and $\gamma$-tocopherol levels were equalised. Importantly, the present study's experimental diet was formulated incorporating whole walnuts as a unit, i.e. as whole food. Walnuts contain multiple ingredients that have been reported to reduce cancer risk or growth rate ${ }^{(16-20)}$. The use of whole walnuts in the study diets was adopted in response to the contrasting results found when specific compounds were tested alone compared with as part of a food. A recent example is the report of $\mathrm{Hsu}$ et $a l^{(21)}$ who found that an isoflavone-rich soya extract induced apoptosis in human PCa cells without inducing apoptosis in benign prostate hyperplasia cells in contrast to specific soya isoflavones at the same levels as the extract induced apoptosis in both cell types ${ }^{(21)}$. An increasing number of such studies suggest that foods exert effects beyond those of any one constituent ${ }^{(22)}$. The present work represents the first study examining the effects of whole walnut-containing diet on PCa in an animal model.

\section{Materials and methods}

Animals

C57/BL6 TRAMP $\times$ C57BL/6 male PB-Tag Line 8247 transgene mice were purchased from Jackson Laboratories. Mice (6-7 weeks old) were fed a laboratory rodent diet (no. 5001, Ralston Purina) for 1 week, then weighed and randomised by weight into three experimental dietary groups (ten to fifteen mice per group) for each of three feeding durations $(9,18$ and 24 weeks of ad libitum feeding). The mice were housed four to a wire-bottomed cage with activity enhancements (e.g. toys and tunnels) in an environmentally controlled room (20$22^{\circ} \mathrm{C}, 60 \%$ relative humidity, $12 \mathrm{~h}$ light $-12 \mathrm{~h}$ dark cycle). Institutional and national guidelines for the care and use of animals were followed and all experimental procedures involving animals were approved by the Animal Care and Use Committee, USDA Western Regional Research Center, Albany, CA, USA.

\section{Diets}

Semisynthetic American Institute of Nutrition (AIN)-93M ${ }^{(23)}$ based diets were produced using either shelled whole walnut halves (English walnuts (Juglans regia), California Walnut Commission) ground to $3 \mathrm{~mm}$ size (WW) or tocopherol-stripped soyabean oil (HF) as fat sources (Table 1). The WW diets incorporated $155 \mathrm{~g}$ of $\mathrm{WW} / \mathrm{kg}$ diet, the equivalent of $80 \mathrm{~g}$ (approximately 3 ounces) of walnuts/d in humans, to give $20.5 \%$ of energy from fat, while the HF diet used $100 \mathrm{~g}$ of tocopherolstripped soyabean oil/kg diet supplying $20 \cdot 7 \%$ of energy from fat. A third diet, the low-fat (LF) diet, used a lower amount of stripped soyabean oil to supply $8.7 \%$ of energy from fat. The two HF provided a closely matched fatty acid profile and the HF and WW diets were equivalent in terms of fat, protein, carbohydrate, fibre, minerals, vitamins as well as $\alpha$ - and $\gamma$-tocopherol levels $(7.5$ and $62 \mathrm{mg} / \mathrm{kg}$ of diet, $\alpha$ and $\gamma$-tocopherol, respectively). Differing amounts of pure $\mathrm{D}-\boldsymbol{\alpha}-$ and $\mathrm{D}-\boldsymbol{\gamma}$-tocopherol (Acros Organics) were added (Table 1) to the walnut and the LF diets to adjust the $\alpha$ - and $\gamma$-tocopherol levels. The energy content of the LF diet was adjusted by the increased carbohydrate content. All diets were made mineral-replete by use of the Mineral mix (AIN93M-MX). The relatively high Se content of walnuts increased the Se content of the WW to $0.7 \mathrm{mg} / \mathrm{kg}$ compared to $0.2 \mathrm{mg} / \mathrm{kg}$ in the other diets. All other nutrients were provided in all diets at concentrations that met or exceeded the minimum recommendations of the $\mathrm{AIN}^{(23)}$.

Table 1. Diet composition (weight added/kg diet) by diet (low-fat (LF), high-fat (HF) and whole walnut (WW))

\begin{tabular}{|c|c|c|c|}
\hline Ingredient added & LF & $\mathrm{HF}$ & WW \\
\hline Fat content & 40 & 100 & 100 \\
\hline English walnuts & - & - & 155 \\
\hline Soyabean oil (no additives) & 40 & 100 & - \\
\hline Saturated $(\%$ of fat) & 9.9 & $9 \cdot 9$ & $5 \cdot 7$ \\
\hline Monounsaturated (\% of fat) & $14 \cdot 8$ & $14 \cdot 8$ & 14.4 \\
\hline Polyunsaturated, total (\% of fats) & $37 \cdot 6$ & $37 \cdot 6$ & 39.9 \\
\hline $18: 3 n-3: 18: 2 n-6$ & 0.2 & 0.2 & 0.24 \\
\hline$\alpha$-Tocopherol $/ \gamma$-tocopherol content & \multicolumn{3}{|c|}{$7.5 \mathrm{mg} / 62 \mathrm{mg}$} \\
\hline$\gamma$-Tocopherol & $38.4 \mathrm{mg}$ & - & $31.5 \mathrm{mg}$ \\
\hline$\alpha$-Tocopherol & $4.8 \mathrm{mg}$ & - & $6.5 \mathrm{mg}$ \\
\hline Maize starch & 465.7 & $405 \cdot 7$ & 385.8 \\
\hline Casein ( $>85 \%$ protein) & 140 & 140 & 116 \\
\hline Dextrinised maize starch (90-94\% tetrasaccharides) & 155 & 155 & 155 \\
\hline Sucrose & 100 & 100 & 100 \\
\hline Fibre & 50 & 50 & $39 \cdot 6$ \\
\hline Mineral mix (AIN-93M-MX) & 35 & 35 & 35 \\
\hline Vitamin mix (AIN-93-VX; vitamin E free) & 10 & 10 & 10 \\
\hline L-Cys & $1 \cdot 8$ & 1.8 & $1 \cdot 8$ \\
\hline
\end{tabular}

AIN, American Institute of Nutrition. 
The diets were fed ad libitum with feed consumption recorded two times per week. Animals were weighed every week and then were killed by anaesthesia overdose/ diaphragm puncture at the pre-planned time sampling intervals (i.e. 9, 18 and 24 weeks). Cardiac puncture was used to obtain blood; and plasma was isolated and stored as multiple aliquots at $-80^{\circ} \mathrm{C}$ until analysis. Prostate genitourinary intact (GUI) tract, i.e. bladder, seminal vesicles, coagulating and ampulary glands were removed and weighed. GUI weight correlates closely with other measures of prostate tumour growth $^{(24)}$ and its use reduces time delays and minimises tissue identification ambiguities that arise during dissection as a result of the increasingly disturbed prostate tissue architecture over time in the TRAMP mouse. The GUI were bisected along the long axis of the urethra and then one portion flash-frozen in liquid $\mathrm{N}_{2}$ and the other stored in $10 \%$ buffered formalin. Finally, whole liver was removed, flashfrozen in liquid $\mathrm{N}_{2}$ and stored at $-80^{\circ} \mathrm{C}$ until analysis.

\section{Prostate pathology}

For histological evaluation, prostate tissues were fixed in $10 \%$ buffered formalin, processed and embedded in paraffin. Tissue sections ( $4 \mu \mathrm{m}$ thick) were cut and stained with haematoxylin/eosin and the histology interpreted by Cardiff and colleagues ${ }^{(25)}$

\section{Plasma analysis}

Multiplex microbead suspension arrays (Luminex Corporation) were used to simultaneously assay several plasma analytes. Both multiple-analyte (insulin, C-reactive protein, adiponectin, leptin, tissue plasminogen activator inhibitor 1 (tPAI-1), resistin) and single-analyte microbead assays for insulin-like growth factor 1 (IGF-1) and glucagon were used according to the manufacturer's instructions (Millipore).

Plasma lipoprotein cholesterol levels were analysed using size-exclusion HPLC coupled to post-column cholesterol quantification as previously described ${ }^{(26)}$.

\section{Liver metabolomic analysis}

Liver tissue samples ( $n 5$ ) at both the 9- and 18-week time points were selected at random from the HF, WW and LF diet groups and analysed using mView, a metabolomic profiling system (Metabolon, Inc.). Compounds were identified via matching with metabolomic library entries of purified standards.

\section{Statistical analysis}

Data collected were entered into Excel spreadsheets for each mouse; and statistical analysis was performed using ANOVA (JMP software v9.0 for Macintosh; SAS, Inc.) after testing for equal variances ${ }^{(27)}$ and then group mean differences were tested, with $P<0.05$ considered significant.

The effects of diet on whole animal weight and prostate weight time-course were estimated in a mixed-effect repeated-measures growth curve model using a square-root time scale transformation to allow for slowing of growth rates with maturity ${ }^{(28)}$. The standard HF diet was chosen as a reference, and pre-planned comparisons were made between the LF diet and the HF diet, and between the WW and HF diets using SAS/STAT ${ }^{\circledR}$ software or R software; and all hypothesis tests were two-sided at $P<0.05$. Prostate weight measures were naturally log-transformed and were with an assumed common mean size at 9 weeks before use in the mixed-effect repeated-measures growth curve model.

Metabolomics data were assessed using Welch's two-sample $t$ test. $Q$ values were computed, but no absolute cut-off was set in order to maximise the power to detect differentially regulated metabolites; and those ratios having a $P<0.05$ were considered significant.

\section{Results}

As anticipated, histological evaluation at the later sampling times found progressive changes in GUI histology. Assessment of the histology documented the presence of a variety of tissue derangements (e.g. engorged seminal vesicles, diffuse atypical prostate hyperplasia); and the numbers of animals with prostate tumours found are presented in Table 2. Upon statistical analysis using exact logistic regression, duration of time on the diet but not diet composition was related significantly with the number of mice with any tumour $(P<0 \cdot 0001)$ as well as for the number of mice with only phylloides prostate tumours. Using the same analysis for the number of mice but restricting it to mice with only neuroendocrine (NE) prostate tumours, both diet composition and time on the diet approached significance $(P=0.0744$ and $P=0.0765$, respectively).

Fig. 1 presents the overall body weight data, while Fig. 2 presents the prostate weight data, i.e. GUI weights over time. GUI weight at the 9 and 24 weeks sampling time points did not show significant differences by diet. However, GUI prostate weights at 18 weeks did show significant

Table 2. Prostate tumour numbers and types by histopathology by diet group (low-fat (LF), high-fat (HF) and whole walnut (WW))

\begin{tabular}{|c|c|c|c|c|c|c|c|c|c|}
\hline \multirow[b]{3}{*}{ Week } & \multicolumn{9}{|c|}{ Diet } \\
\hline & \multicolumn{3}{|c|}{ LF } & \multicolumn{3}{|c|}{$\mathrm{HF}$} & \multicolumn{3}{|c|}{ WW } \\
\hline & 9 & 18 & 24 & 9 & 18 & 24 & 9 & 18 & 24 \\
\hline Total tumours/total animals & $0 / 9$ & $0 / 8$ & $9 / 10$ & $2 / 11$ & $5 / 9$ & $10 / 15$ & $2 / 9$ & $4 / 12$ & $\begin{array}{c}6 / 8 \\
6 \mathrm{PHY}\end{array}$ \\
\hline Tumour type & 0 & 0 & $3 \mathrm{NE}, 6 \mathrm{PHY}$ & $2 \mathrm{NE}$ & $3 \mathrm{NE}, 2 \mathrm{PHY}$ & $4 \mathrm{NE}, 6 \mathrm{PHY}$ & 1NE, $1 \mathrm{PHY}$ & $4 \mathrm{PHY}$ & $6 \mathrm{PHY}$ \\
\hline
\end{tabular}

NE, neuroendocrine tumour; PHY, phylloides tumour. 


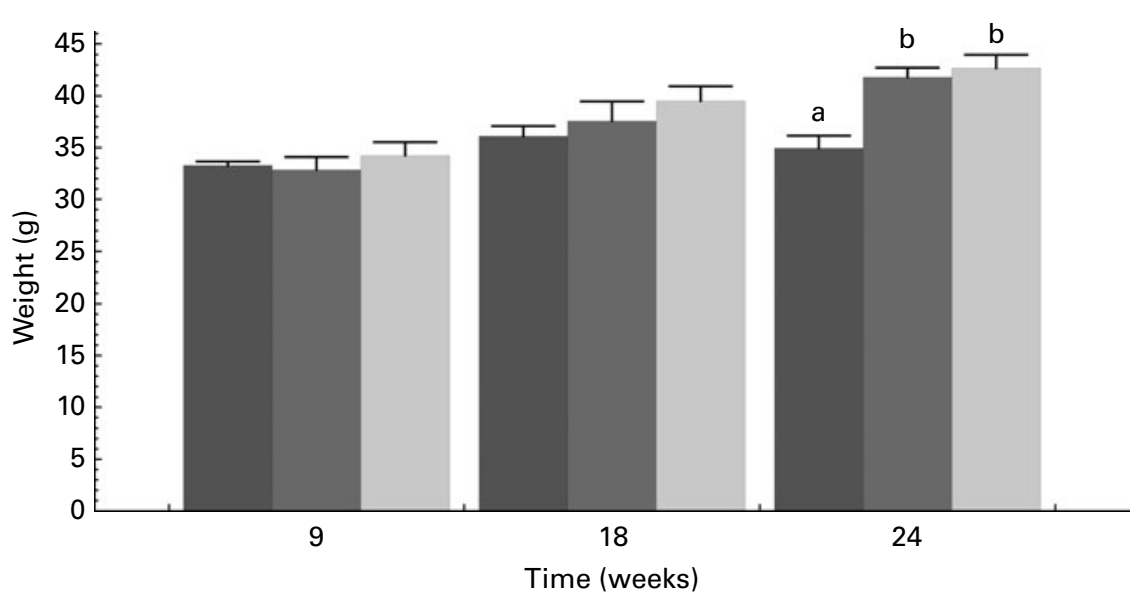

Fig. 1. Body weight for the different diet groups (low-fat $(\square)$, high-fat $(\square)$ and whole walnut $(\square)$ ) at the different sampling times. Values are means with SEM represented by vertical bars. ${ }^{a, b}$ Mean values within the same sampling time with unlike letters were significantly different $(P<0 \cdot 05)$.

differences by diet (Fig. 2) as the LF and WW diet groups' GUI prostate weights were markedly and statistically significantly lower than the HF diet group's GUI prostate weight but did not differ from each other. GUI weights are presented (Fig. 2), but both GUI weight and GUI weight/body weight gave the same results upon statistical analysis. A two-way ANOVA analysis of GUI weight data found that duration of time on the diet reached statistical significance $(P<0 \cdot 05)$.

The effect of diet on whole animal rate of weight gain was analysed using a mixed-effect repeated-measures growth curve model. The analysis revealed that whole body growth rate for the WW diet animals did not differ from that of the HF diet animals $(4.75 v .4 .81 \mathrm{~g}$ /square root (weeks); $P=0.84$ ), but that whole body growth rate of the LF diet animals was about $20 \%$ lower $(3.7 \mathrm{~g} /$ sqrt (weeks); $P=0.001)$ than that of the HF and WW diet animals. In contrast to the lack of effect on body weight, analysis of prostate weight gain using a mixed-effect growth curve model found that the WW diet animal's prostate growth $(8(\operatorname{sem} 2 \cdot 8) \% /$ week $)$ was reduced by $28 \%(P<0 \cdot 015)$ relative to that of the HF diet animals (11 (sEm 2.5)\%/week). The prostate weight gain ( 8 (SEM $2 \cdot 8$ ) \%/week) in the LF diet animals was also reduced by $28 \%(P<0 \cdot 015)$.

Plasma IGF-1 (Fig. 3) differed by diet group at 18 weeks. IGF1 was lower $(P<0.05)$ in the LF and WW diet animals compared to the HF diet animals, but the WW and LF diet animals' IGF-1 levels did not differ from each other. Plasma resistin was lower $(P<0.05)$ in the 18-week WW and LF animals compared to the HF diet animals (Table 3), but the WW and LF diet animals' resistin levels did not differ from each other. Other plasma analytes, i.e. adiponectin, insulin, C-reactive protein and leptin showed a trend towards altered levels with diet group (Table 3), but all insulin failed to achieve statistical significance. LF diet insulin differed only from HF diet at 18 weeks.

While plasma total cholesterol at 18 weeks did not differ between the HF and WW diets, at 18 weeks both WW diet group LDL-cholesterol and LDL-cholesterol:HDL-cholesterol ratios were significantly lower when compared to the HF diet but not compared to the LF diet (Table 4).

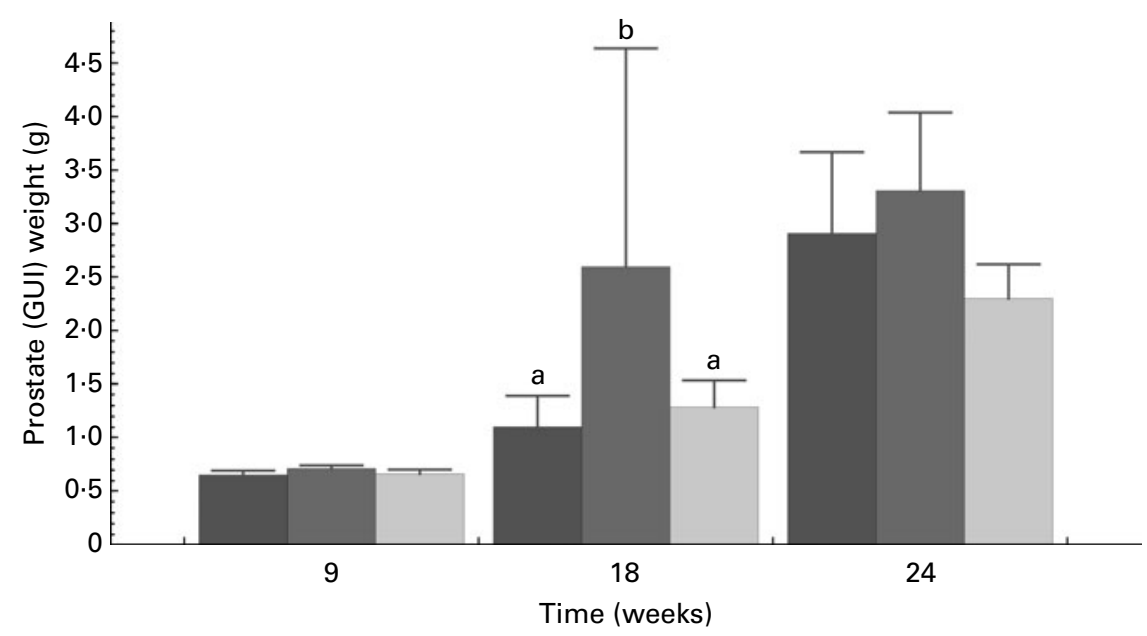

Fig. 2. Prostate weight as genitourinary tract intact (GUI) for the different diet groups (low-fat ( $\square$ ), high-fat ( $\square$ ) and whole walnut ( $\square)$ ) at the different sampling times. Values are means with SEM represented by vertical bars. ${ }^{a, b}$ Mean values within the same sampling time with unlike letters were significantly different $(P<0.05)$ 


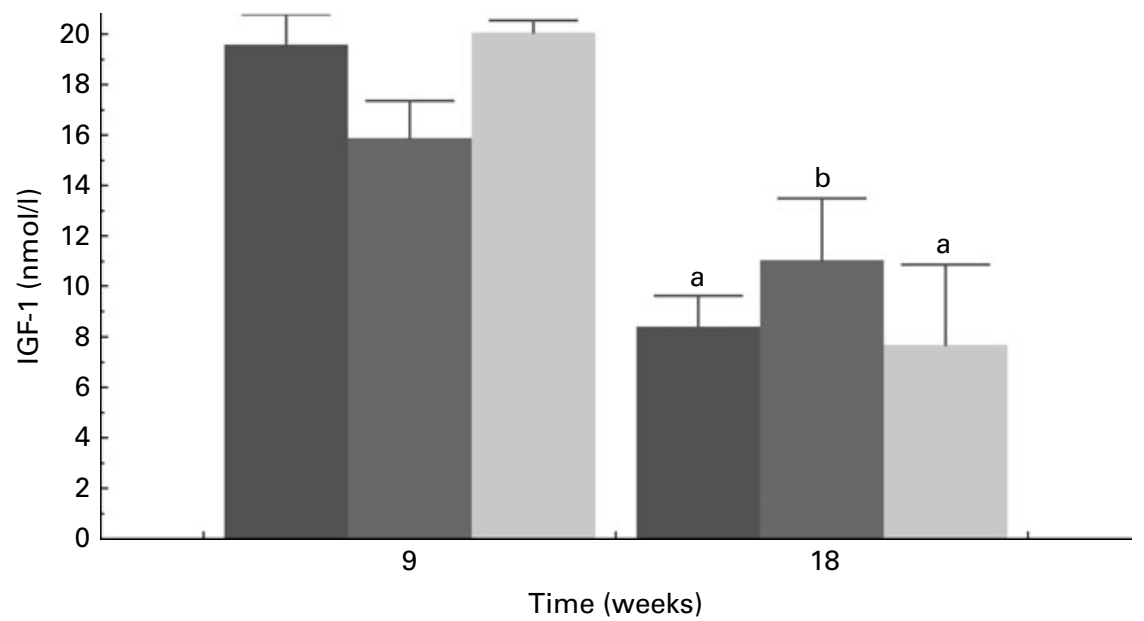

Fig. 3. Plasma insulin-like growth factor 1 (IGF-1) levels are presented for the different diet groups (low-fat ( $\square)$, high-fat ( $\square)$ and whole walnut $(\square)$ ) at 9 weeks, and 18 weeks of ad libitum feeding. Values are means with SEM represented by vertical bars. ${ }^{a, b}$ Mean values within the same sampling time with unlike letters were significantly different $(P<0.05)$.

Metabolomic analysis of the TRAMP mouse livers found twenty and twenty-six metabolites among the 266 identified named biochemicals, showing significant $(P \leq 0.05)$ differences in levels between the HF and WW diet groups at 9 and 18 weeks, respectively (Table 5, see the supplementary table for metabolites identified and diet-related differences, supplementary material for this article can be found at http://www.journals.cambridge.org/bjn). With respect to the lipid-related metabolites identified and found to differ by diet, $n$-3 fatty acids along with $n$ - 3 fatty acid-containing lysolipids (i.e. linoleoyl-glycerophosphoethanolamine) were elevated in WW liver, while arachidonate, an n-6 fatty acid along with $n$ - 6 fatty acid-containing lysolipids were decreased compared to HF liver. In addition, hepatic sphingosine content was over 2-fold lower in WW mice compared to that of the HF diet group at 18 weeks and WW livers had significantly increased 9- and 13-hydroxy-octadecadienoic acids.

\section{Discussion}

High fat intake, particularly saturated fat intake, has been associated with CVD, other metabolic diseases and some forms of cancer including $\mathrm{PCa}^{(29)}$. Walnuts, like other nuts, are typically consumed whole, with a majority of their energy coming from fat. Although high in fat, walnut fats are mainly polyunsaturated and the effects of consuming walnuts on PCa are unclear ${ }^{(10)}$ and was the focus of this study. The results of the present study demonstrate that whole walnuts have a beneficial effect in an animal model of PCa. The walnut diet, irrespective of its high fat content, resulted in notable and statistically significant decreased GUI weights and rate of tumour weight gain. The present study results parallel those of Adhami and colleagues ${ }^{(30)}$ who demonstrated that green tea also reduced prostate GUI weight in TRAMP mice, which they showed correlated closely with prostate tumour burden and occurrence. This congruence lends support to the conclusion that the WW diet effect is a result of walnut diet-induced decreased prostate tumour growth rate and reduced prostate tumour size.

The TRAMP model has been much criticised as a NE-cancer model with questionable relevance to human $\mathrm{PCa}$; and more recently, the C57BL/6 TRAMP mouse has been suggested to actually represent a mixed model of prostate carcinogenesis $^{(31,32)}$. One tumour process is the phylloides tumour which is an androgen-driven glandular prostate atypical hyperplasia in the dorsolateral prostate (DLP) lobes while the other is NE-cancer in the ventral prostate (VP) lobes. This is of importance, as the TRAMP DLP lesion progression

Table 3. Mouse plasma analyte levels (nM) either at 9 and 18 weeks or at 18 weeks-only time points by diet group (low-fat (LF), high-fat (HF) and whole walnut (WW))

(Number of animals, mean values with SEM)

\begin{tabular}{|c|c|c|c|c|c|c|c|c|c|c|}
\hline \multirow[b]{2}{*}{ Analyte } & \multirow[b]{2}{*}{ Weeks } & \multicolumn{3}{|c|}{ LF diet } & \multicolumn{3}{|c|}{ HF diet } & \multicolumn{3}{|c|}{ WW diet } \\
\hline & & $n$ & Mean & SEM & $n$ & Mean & SEM & $n$ & Mean & SEM \\
\hline Resistin & 9 & 5 & 0.19 & 0.03 & 4 & 0.21 & 0.03 & 8 & 0.21 & 0.02 \\
\hline Insulin & 9 & 4 & $65 \cdot 6$ & $36 \cdot 7$ & 2 & $75 \cdot 2$ & $23 \cdot 8$ & 6 & $40 \cdot 6$ & 5.9 \\
\hline Resistin & 18 & 9 & $0.21^{a}$ & 0.02 & 8 & $0.13^{b}$ & 0.02 & 11 & $0 \cdot 16^{b}$ & 0.02 \\
\hline Insulin & 18 & 8 & $60 \cdot 2^{a}$ & $10 \cdot 4$ & 8 & $23.8^{\mathrm{b}}$ & 5.5 & 10 & $36 \cdot 6^{\mathrm{a}, \mathrm{b}}$ & $6 \cdot 2$ \\
\hline Adiponectin & 18 & 9 & 0.029 & 0.003 & 8 & 0.048 & 0.010 & 12 & 0.036 & 0.002 \\
\hline CRP & 18 & 9 & 93.0 & $5 \cdot 3$ & 8 & 89.8 & $7 \cdot 1$ & 12 & $84 \cdot 8$ & $3 \cdot 2$ \\
\hline Leptin & 18 & 9 & 1.85 & 1.27 & 8 & 0.29 & 0.07 & 11 & 0.89 & 0.21 \\
\hline
\end{tabular}

CRP, C-reactive protein.

a,b Mean values within a row with unlike superscript letters were significantly different $(P<0.05)$. 
Table 4. Mouse plasma cholesterol levels (mM) in lipoprotein fractions and as total at 18-week time point by diet group (low-fat (LF), high-fat (HF) and whole walnut (WW))

(Mean values with their standard errors)

\begin{tabular}{|c|c|c|c|c|c|c|c|c|c|c|}
\hline \multirow[b]{2}{*}{ Diet } & \multicolumn{2}{|c|}{ Total } & \multicolumn{2}{|c|}{ VLDL } & \multicolumn{2}{|c|}{ LDL } & \multicolumn{2}{|c|}{$\mathrm{HDL}$} & \multicolumn{2}{|c|}{ LDL:HDL } \\
\hline & Mean & SEM & Mean & SEM & Mean & SEM & Mean & SEM & Mean & SEM \\
\hline LF & 2.04 & 0.30 & 0.05 & 0.005 & $0.28^{b}$ & 0.05 & 1.72 & 0.26 & $0.16^{b}$ & 0.03 \\
\hline $\mathrm{HF}$ & $2 \cdot 70$ & 0.46 & 0.05 & 0.005 & $0.66^{\mathrm{a}}$ & 0.12 & 1.98 & 0.34 & $0.32^{\mathrm{a}}$ & 0.03 \\
\hline WW & 2.44 & 0.13 & 0.06 & 0.005 & $0.36^{b}$ & 0.05 & 2.01 & 0.09 & $0.18^{\mathrm{b}}$ & 0.02 \\
\hline
\end{tabular}

${ }^{\mathrm{a}, \mathrm{b}}$ Mean values within a row with unlike letters were significantly different $(P<0.05)$.

is more characteristic of human prostate epithelial adenocarcinogenesis and the majority of human PCa are adenocarcinomas. Conversely, NE-like carcinomas lack androgen receptor, express synaptophysin and belong to a distinct lineage ${ }^{(31,32)}$. While the present study did not find a statistically significant diet effect in terms of tumour occurrence, the tumour type showed some degree of diet dependence, as NE tumours in the walnut-fed group were nearly absent. That the walnut diet group TRAMP mice prostates are relatively free of NEcancer while also showing reduced size may therefore reflect the walnut-related effects on phylloide tumours, as these DLP lesions account for most of TRAMP prostate weight gains ${ }^{(32)}$ in combination with possible effects on prostate NE tumours. Given the nature of the genetic construct driving the TRAMP tumour model, tumour suppression by walnuts was unlikely to occur, which makes the finding of slowed growth of importance. The specificity of the walnut diet's effects with respect to tumour lineages and prostate lobes affected awaits future study.

This study's results further suggest that PCa growth does not appear to be a function of the level of dietary fat, as both the WW and HF diets had the same fat level. Freedland and coworkers have recently reported that tumour growth was not associated with dietary fat content, using a mouse PCa xenograft model $^{(12,33)}$. A similar conclusion regarding the relationship of fat content to cancer growth has been reported recently for pancreatic cancer ${ }^{(34)}$. The present study's WW diet not only had the same fat content as the HF diet, but were substantially similar in their fatty acid profiles removing that as a factor for the dietassociated effects noted. In addition, the absence of similar effects despite the identical levels of $\alpha$ - and $\gamma$-tocopherol supplied by both the WW and the HF diets eliminates these tocopherols as a factor in the differences.

The walnut diet differs in Se (approximately $0.9 \mathrm{mg} / \mathrm{kg}$ diet in WW $v$. approximately $0.2 \mathrm{mg} \mathrm{Se} / \mathrm{kg}$ diet in the $\mathrm{HF}$ and LF diets), as English walnuts are relatively high in Se (United States Department of Agriculture National Nutrient Database for Standard Reference, Release 24). However, the modest levels of Se for any of the study diets tested makes it difficult to ascribe the differences to Se, as much higher Se levels $(3.0 \mathrm{mg} \mathrm{Se} / \mathrm{kg}$ diet) were used in studies of Se-related effects in the TRAMP prostate model along with other rodent models ${ }^{(35,36)}$. Added difficulty in linking Se levels to effects arises from the recent report that Se-related effects in mice depend upon composition of the basal diet consumed along with the differences linked to the form of Se tested ${ }^{(35,36)}$.
Finally, the reduced tumour growth in the WW diet cannot be explained by differences in energy bioavailability between the diets, as there were no differences in body weights and body-weight gains between WW when compared to the HF diet-fed mice.

Diet and increased PCa have been linked via increased IGF- ${ }^{(37)}$. The present study found that IGF-1 in WW diet mice at 18 weeks was reduced and this was associated with smaller tumour size and slower tumour growth rate. The study findings of reduced IGF-1 and smaller tumour size/ growth are in agreement with other PCa studies that have either specifically modulated or monitored IGF-1 in TRAMP mice $^{(30,31,38)}$. Elevated IGF-1, along with elevated insulin, has been reported to increase the risk of PCa via increased cell proliferation and cell survival ${ }^{(39)}$. The influence of IGF-1 levels on prostate tumour growth is highlighted by the contrast between both animal survival and prostate tumour cell

Table 5. Liver metabolomic results

\begin{tabular}{|c|c|c|}
\hline & 9 weeks & 18 weeks \\
\hline \multicolumn{3}{|l|}{ Biochemical } \\
\hline Campesterol & $0.29^{\star}$ & 0.40 \\
\hline Sphingosine & 0.56 & $0.44^{*}$ \\
\hline Sphinganine & $0.58^{*}$ & 0.45 \\
\hline Myristate (14:0) & 0.93 & $0.67^{\star}$ \\
\hline 1-Myristoylglycerophosphocholine & $0.49^{*}$ & 1.02 \\
\hline 1-Oleoylglycerophosphocholine & $0.38^{*}$ & 0.78 \\
\hline 1-Palmitoleoylglycerophosphocholine & $0.38^{*}$ & 0.78 \\
\hline 1-Palmitoylglycerophosphoethanolamine & $0.60^{*}$ & 1.09 \\
\hline 1-Palmitoylglycerophosphoinositol & 0.65 & $0.70^{*}$ \\
\hline 2-Oleoylglycerophosphocholine & $0.41^{*}$ & 0.47 \\
\hline 2-Palmitoleoylglycerophosphocholine & $0.42^{*}$ & 0.60 \\
\hline 2-Palmitoylglycerophosphoethanolamine & $0.41^{*}$ & 0.84 \\
\hline 2-Hydroxystearate & $1 \cdot 12$ & $1.96^{*}$ \\
\hline$\alpha$ - or $\gamma$-Linolenate $(18: 3 n-3$ or 6$)$ & 1.61 & $2 \cdot 17^{\star}$ \\
\hline \multicolumn{3}{|l|}{$n-6$ Fatty acid-related } \\
\hline Arachidonate $(20: 4 n-6)$ & $0.73^{\star}$ & $0.80^{*}$ \\
\hline 2-Arachidonoylglycerophosphocholine & $0.41^{*}$ & 0.61 \\
\hline Linoleate $(18: 2 n-6)$ & $1 \cdot 11$ & $1.43^{*}$ \\
\hline 1-Linoleoylglycerophosphoethanolamine & 1.49 & $2 \cdot 50^{*}$ \\
\hline 2-Linoleoylglycerophosphoethanolamine & 1.22 & $2 \cdot 08^{*}$ \\
\hline 13-HODE + 9-HODE & 1.89 & $1.82^{*}$ \\
\hline \multicolumn{3}{|l|}{$n-3$ Fatty acid-related } \\
\hline Docosapentaenoate ( $n-3$ DPA; $22: 5 n-3$ ) & 1.47 & $1.43^{*}$ \\
\hline 2-Docosahexaenoylglycerophosphocholine & $0.58^{*}$ & 0.74 \\
\hline $\begin{array}{l}\text { 2-Docosahexaenoylglycero } \\
\text { phosphoethanolamine }\end{array}$ & $0.54^{\star}$ & 0.79 \\
\hline EPA $(20: 5 n-3)$ & 1.23 & $1.89^{*}$ \\
\hline
\end{tabular}

HODE, hydroxy-octadecadienoic acid.

${ }^{\star}$ Statistically significant lipid related metabolite changes $(P<0.05)$ are presented as the ratio of the whole walnut diet or the high fat diet abundance at 9 and 18 weeks. 
growth in studies that altered IGF-1 levels. Studies with increased survival and decreased tumour cell growth were those treatments that lowered IGF-1 compared to those where IGF-1 was unaffected ${ }^{(12,33,40,41)}$. Finally, Chen et al. ${ }^{(42)}$ have reported that enterolactone, a major metabolite of plant-based lignans inhibits PCa cell growth in vitro and does so, at least in part via inhibition of IGF-1/IGF-1R signalling.

Recent clinical studies targeting the IGF-1R receptor alone or in combination with chemotherapy have shown potent PCa growth inhibition ${ }^{(43)}$. However, the theoretical constructs linking prostate tumour growth and IGF-1 signalling axis have proven difficult to rationalise as the effects of genetically modifying IGF-1 levels or IGF-1 signalling pathways on PCa in TRAMP mice have given mixed results ${ }^{(44-46)}$. The accelerated lesion progression and more aggressive phenotype upon knockout of prostate epithelial cell IGF-1R in the TRAMP model has suggested that there are different thresholds for prostate IGF-1 signalling and that complete IGF-1R knockout cannot recapitulate diminished but intact IGF-1/IGF-1R signalling ${ }^{(46)}$. Interestingly, Wang et al. $^{(31)}$ suggested that Se inhibition of PCa tumour growth might be via effects on both IGF-1 ligand and receptor. Again, the present study's WW and HF diets' identical $\alpha$ - and $\gamma$-tocopherol, fat content and substantially similar fatty acid profiles removes these as factors for the IGF-1 effects noted in this study. The extent to which changes seen in the present study are from direct effects on the prostate IGF-1 axis, as reported in the case of green tea $^{(30)}$ and silibinin $^{(47)}$, remains to be further explored.

Although earlier reports have suggested that altered resistin levels are not associated with $\mathrm{PCa}^{(48)}$, recent results from Kim and colleagues have suggested that they are elevated in $\mathrm{PCa}^{(49)}$ and stimulate PCa growth ${ }^{(50)}$. The present study revealed that mice on the WW diet had reduced levels of resistin compared to the HF diet group animals, a similar pattern to that found for IGF-1 levels. Other plasma analytes showed a trend towards altered levels with diet, but none of these achieved statistical significance. For example, low levels of adiponectin have been linked to $\mathrm{PCa}^{(51,52)}$ and $\mathrm{WW}$ diet mice at 18 weeks had tended to have increased adiponectin levels $(P=0.051)$ compared to the HF diet. Aronis et al. ${ }^{(53)}$ have very recently reported that walnut consumption acutely increases plasma adiponectin in obese patients. The connection between changes in PCa growth and the effects of altered resistin and, potentially, adiponectin remains to be further clarified.

Mice in the WW group exhibited significant changes in plasma cholesterol metabolism, specifically decreased LDLcholesterol and altered LDL-C:HDL-C ratios relative to those in the HF diet. Circulating cholesterol has been reported to promote prostate tumour growth ${ }^{(54,55)}$. LNCaP PCa cells when cultured in vitro with decreased endogenous cholesterol availability increase their LDL receptor activity, presumably in order to acquire cholesterol required to support their growth $^{(56)}$. Recently, using the same TRAMP mouse model, a high-fat (60\% of energy as fat) in combination with $0 \cdot 2 \%$ cholesterol diet (i.e. a 'Western' diet) was shown to elevate plasma cholesterol, alter LDL-C:HDL-C ratios and accelerate prostate tumour onset and progression ${ }^{(55)}$. Specific lipoprotein classes such as LDL and HDL are associated with delivery or removal of cell cholesterol ${ }^{(57)}$ and have been linked to $\mathrm{PCa}^{(58)}$. The changes noted with diet in the plasma markers studied are echoed in the liver metabolomics results. Interestingly, despite the similarities in fats between the two diets, the WW diet group liver metabolome showed elevations of $n-3$ fatty acid-related lipids while $n-6$ fatty acid-related lipids decreased compared to the HF diet group livers. These changes are probably of significance as $n$ - 3 fatty acid metabolism and their effects in PCa have been of high interest ${ }^{(3-7)}$. In addition, the diet-driven changes noted in the metabolites in the liver are noteworthy, given the liver's central role in both IGF-1 and in plasma lipid and lipoprotein metabolism ${ }^{(59)}$. However, the linkage of PCa and other cancers with specific fatty acids is proving to be very complex. The $n-3$ fatty acid, DHA, has been recently reported as positively associated with high-grade PCa, contrary to what the study authors had hypothesised $^{(60)}$. In addition, Hardman and colleagues ${ }^{(14)}$ have just reported that while consumption of walnuts significantly reduced the incidence, multiplicity and size of mammary tumours, a cancer that shares multiple characteristics with $\mathrm{PCa}^{(61)}$, the $n-3$ content alone did not account for the extent of tumour suppression due to the walnut diet.

In summary, the present study found that whole walnuts fed as part of a HF diet (i.e. the WW diet) reduce TRAMP mouse PCa tumour growth and size. The walnut diet-consuming animals also showed declines in plasma IGF-1, resistin as well as LDL, elevations of which have all been linked to tumour growth. The study results further suggest that the walnut effects are not due to their specific fatty acid or tocopherol content. The differences found between the effects of the whole walnut and the control HF diet when coupled with the disappointing $\alpha$-tocopherol chemopreventative clinical trials emphasises that whole foods and complete diets probably exert effects beyond those of an individual constituent $^{(6,22,62)}$. Moreover, the disparate diet effects observed at the same elevated dietary fat intake suggest that total dietary fat intake is not strongly linked to PCa tumour biology. The study results show that excluding walnuts based on their fat content is unjustified and that consumption of walnuts, already linked to reduced CVD risk ${ }^{(29)}$, will probably reduce the risk of $\mathrm{PCa}$ in humans. Finally, these findings point to the need for studies using whole foods such as walnuts to truly assess their effects on PCa and to identify effective, food-based chemoprevention diets for PCa as well as other cancers.

\section{Acknowledgements}

All authors participated in the design of the present study. Diets were produced and animal husbandry supervised by W. Y. Tissue and plasma samples were obtained by P. A. D., K. G., W. Y. and H. K. Plasma analytes were determined by I. H. K. and W. Y. Data analysis was performed by P. A. D., except as noted herein. The initial manuscript draft was written by P. A. D., critically revised by C. E. C., W. Y., H. K., I. H. K., V. T. V. and K. G. The final version was assembled by P. A. D. and all authors read and approved the final manuscript. 
This study was supported by a research grant to the University of California, Davis from the California Walnut Board. Statistical analysis support was provided by grant number UL1 RR024146 from the National Center for Research Resources (NCRR), a component of the National Institutes of Health (NIH), and NIH Roadmap for Medical Research. The manuscript's contents are solely the responsibility of the authors and do not necessarily represent the official view of the NCRR or NIH. The authors have no conflicts of interest to declare.

\section{References}

1. Jemal A, Siegel R, Xu J, et al. (2010) Cancer statistics. CA Cancer J Clin 60, 277-300.

2. Escobar EL, Gomes-Marcondes MC \& Carvalho HF (2009) Dietary fatty acid quality affects AR and PPARgamma levels and prostate growth. Prostate 69, 548-558.

3. Brown MD, Hart C, Gazi E, et al. (2010) Influence of omega6 PUFA arachidonic acid and bone marrow adipocytes on metastatic spread from prostate cancer. $\mathrm{Br} J$ Cancer 102, 403-413.

4. Berquin IM, Min Y, Wu R, et al. (2007) Modulation of prostate cancer genetic risk by omega- 3 and omega- 6 fatty acids. J Clin Invest 117, 1866-1875.

5. Reese AC, Fradet V \& Witte JS (2009) Omega-3 fatty acids, genetic variants in COX-2 and prostate cancer. $J$ Nutrigenet Nutrigenomics 2, 149-158.

6. Kristal AR, Arnold KB, Neuhouser ML, et al. (2010) Diet, supplement use, and prostate cancer risk: results from the prostate cancer prevention trial. Am J Epidemiol 172, 566-577.

7. Vissapragada S, Ghosh A, Ringer L, et al. (2010) Dietary n-3 polyunsaturated fatty acids fail to reduce prostate tumorigenesis in the PB-ErbB-2x Pten $(+/-)$ preclinical mouse model. Cell cycle 9, 1824-1829.

8. Szymanski KM, Wheeler DC \& Mucci LA (2010) Fish consumption and prostate cancer risk: a review and metaanalysis. Am J Clin Nutr 92, 1223-1233.

9. Hori S, Butler E \& McLoughlin J (2011) Prostate cancer and diet: food for thought? BJU Int 107, 1348-1359.

10. Spaccarotella KJ, Kris-Etherton PM, Stone WL, et al. (2008) The effect of walnut intake on factors related to prostate and vascular health in older men. Nutr J 7, 13

11. Freedland SJ \& Aronson WJ (2009) Dietary intervention strategies to modulate prostate cancer risk and prognosis. Curr Opin Urol 19, 263-267.

12. Lloyd JC, Antonelli JA, Phillips TE, et al. (2010) Effect of isocaloric low fat diet on prostate cancer xenograft progression in a hormone deprivation model. J Urol 183, 1619-1624.

13. Hardman WE \& Ion G (2008) Suppression of implanted MDA-MB 231 human breast cancer growth in nude mice by dietary walnut. Nutr Cancer 60, 666-674.

14. Hardman WE, Ion G, Akinsete JA, et al. (2011) Dietary walnut suppressed mammary gland tumorigenesis in the C(3)1 TAg mouse. Nutr Cancer 63, 960-970.

15. Nguewa PA \& Calvo A (2010) Use of transgenic mice as models for prostate cancer chemoprevention. Curr Mol Med 10, 705-718

16. Petrik MB, McEntee MF, Johnson BT, et al. (2000) Highly unsaturated ( $n$ - 3 ) fatty acids, but not alpha-linolenic, conjugated linoleic or gamma-linolenic acids, reduce tumorigenesis in Apc(Min/+) mice. J Nutr 130, 2434-2443.

17. Ju YH, Clausen LM, Allred KF, et al. (2004) $\beta$-Sitosterol, $\beta$-sitosterol glucoside, and a mixture of $\beta$-sitosterol and $\beta$-sitosterol glucoside modulate the growth of estrogen-responsive breast cancer cells in vitro and in ovariectomized athymic mice. J Nutr 134, 1145-1151.

18. Maguire LS, O'Sullivan SM, Galvin K, et al. (2004) Fatty acid profile, tocopherol, squalene and phytosterol content of walnuts, almonds, peanuts, hazelnuts and the macadamia nut. Int J Food Sci Nutr 55, 171-178.

19. Han DH, Lee MJ \& Kim JH (2006) Antioxidant and apoptosisinducing activities of ellagic acid. Anticancer Res $\mathbf{2 6}$ 3601-3606.

20. Berquin IM, Edwards IJ \& Chen YQ (2008) Multi-targeted therapy of cancer by omega-3 fatty acids. Cancer Lett 269 363-377.

21. Hsu A, Bray TM, Helferich WG, et al. (2010) Differential effects of whole soy extract and soy isoflavones on apoptosis in prostate cancer cells. Exp Biol Med (Maywood) 235, 90-97.

22. Jacobs DR Jr, Gross MD \& Tapsell LC (2009) Food synergy: an operational concept for understanding nutrition. $\mathrm{Am} \mathrm{J}$ Clin Nutr 89, 1543S-1548S.

23. Reeves PG, Nielsen FH \& Fahey GC Jr (1993) AIN-93 purified diets for laboratory rodents: final report of the American Institute of Nutrition ad hoc writing committee on the reformulation of the AIN-76A rodent diet. $J$ Nutr 123, 1939-1951.

24. Gupta S, Adhami VM, Subbarayan M, et al. (2004) Suppression of prostate carcinogenesis by dietary supplementation of celecoxib in transgenic adenocarcinoma of the mouse prostate model. Cancer Res 64, 3334-3343.

25. Qi J, Nakayama K, Cardiff RD, et al. (2010) Siah2-dependent concerted activity of HIF and FoxA2 regulates formation of neuroendocrine phenotype and neuroendocrine prostate tumors. Cancer Cell 18, 23-38.

26. Xu R, Yokoyama WH, Irving D, et al. (1998) Effect of dietary catechin and vitamin $\mathrm{E}$ on aortic fatty streak accumulation in hypercholesterolemic hamsters. Atherosclerosis 137, 29-36.

27. Brown MB \& Forsythe AB (1974) Robust tests for equality of variances. J Amer Statist Assoc 69, 364-367.

28. Laird NM \& Ware JH (1982) Random-effects models for longitudinal data. Biometrics 38, 963-974.

29. Kris-Etherton PM, Hu FB, Ros E, et al. (2008) The role of tree nuts and peanuts in the prevention of coronary heart disease: multiple potential mechanisms. J Nutr 138, 1746S-1751S.

30. Adhami VM, Siddiqui IA, Ahmad N, et al. (2004) Oral consumption of green tea polyphenols inhibits insulin-like growth factor-I-induced signaling in an autochthonous mouse model of prostate cancer. Cancer Res 64, 8715-8722.

31. Wang L, Bonorden MJ, Li GX, et al. (2009) Methyl-selenium compounds inhibit prostate carcinogenesis in the transgenic adenocarcinoma of mouse prostate model with survival benefit. Cancer Prev Res (Phila Pa) 2, 484-495.

32. Wang L, Zhang J, Zhang Y, et al. (2011) Lobe-specific lineages of carcinogenesis in the transgenic adenocarcinoma of mouse prostate and their responses to chemopreventive selenium. Prostate 71, 1429-1440.

33. Freedland SJ, Mavropoulos J, Wang A, et al. (2008) Carbohydrate restriction, prostate cancer growth, and the insulin-like growth factor axis. Prostate 68, 11-19.

34. Cheon EC, Strouch MJ, Barron MR, et al. (2011) Alteration of strain background and a high omega- 6 fat diet induces earlier onset of pancreatic neoplasia in EL-Kras transgenic mice. Int J Cancer 128, 2783-2792.

35. Quiner TE, Nakken HL, Mason BA, et al. (2011) Soy content of basal diets determines the effects of supplemental selenium in male mice. $J$ Nutr 141, 2159-2165. 
36. Zhang J, Wang L, Li G, et al. (2011) Mouse prostate proteomes are differentially altered by supranutritional intake of four selenium compounds. Nutr Cancer 63, 778-789.

37. Key TJ (2011) Diet, insulin-like growth factor-1 and cancer risk. Proc Nutr Soc (epublication ahead of print version 3 May 2011).

38. Prasad AS, Mukhtar H, Beck FW, et al. (2010) Dietary zinc and prostate cancer in the TRAMP mouse model. $I$ Med Food 13, 70-76.

39. Pollak M (2008) Insulin and insulin-like growth factor signalling in neoplasia. Nat Rev Cancer 8, 915-928.

40. Bonorden MJ, Rogozina OP, Kluczny CM, et al. (2009) Intermittent calorie restriction delays prostate tumor detection and increases survival time in TRAMP mice. Nutr Cancer 61, 265-275.

41. Thomas JA 2nd, Antonelli JA, Lloyd JC, et al. (2010) Effect of intermittent fasting on prostate cancer tumor growth in a mouse model. Prostate Cancer Prostatic Dis 13, 350-355.

42. Chen LH, Fang J, Sun Z, et al. (2009) Enterolactone inhibits insulin-like growth factor-1 receptor signaling in human prostatic carcinoma PC-3 cells. J Nutr 139, 653-659.

43. Gualberto A \& Pollak M (2009) Clinical development of inhibitors of the insulin-like growth factor receptor in oncology. Curr Drug Targets 10, 923-936.

44. Majeed N, Blouin MJ, Kaplan-Lefko PJ, et al. (2005) A germ line mutation that delays prostate cancer progression and prolongs survival in a murine prostate cancer model. Oncogene 24, 4736-4740.

45. Anzo M, Cobb LJ, Hwang DL, et al. (2008) Targeted deletion of hepatic Igf1 in TRAMP mice leads to dramatic alterations in the circulating insulin-like growth factor axis but does not reduce tumor progression. Cancer Res 68 3342-3349.

46. Sutherland BW, Knoblaugh SE, Kaplan-Lefko PJ, et al. (2008) Conditional deletion of insulin-like growth factor-I receptor in prostate epithelium. Cancer Res 68, 3495-3504.

47. Raina K, Blouin MJ, Singh RP, et al. (2007) Dietary feeding of silibinin inhibits prostate tumor growth and progression in transgenic adenocarcinoma of the mouse prostate model. Cancer Res 67, 11083-11091.

48. Housa D, Vernerova Z, Heracek J, et al. (2008) Serum resistin levels in benign prostate hyperplasia and non-metastatic prostate cancer: possible role in cancer progression. Neoplasma 55, 442-446.
49. Chung WH, Kim TH, Chang IH, et al. (2009) Relation of resistin to prostate cancer differentiation and aggressiveness. Korean J Urol 50, 540-546.

50. Kim HJ, Lee YS, Won EH, et al. (2010) Expression of resistin in the prostate and its stimulatory effect on prostate cancer cell proliferation. BJU Int 108, E77-E83.

51. Housa D, Vernerova Z, Heracek J, et al. (2008) Adiponectin as a potential marker of prostate cancer progression: studies in organ-confined and locally advanced prostate cancer. Physiol Res 57, 451-458.

52. Grosman H, Fabre B, Mesch V, et al. (2010) Lipoproteins, sex hormones and inflammatory markers in association with prostate cancer. Aging Male 13, 87-92.

53. Aronis KN, Vamvini MT, Chamberland JP, et al. (2011) Shortterm walnut consumption increases circulating total adiponectin and apolipoprotein A concentrations, but does not affect markers of inflammation or vascular injury in obese humans with the metabolic syndrome: data from a doubleblinded, randomized, placebo-controlled study. Metabolism (epublication ahead of print version 9 November 2011)

54. Solomon KR, Pelton K, Boucher K, et al. (2009) Ezetimibe is an inhibitor of tumor angiogenesis. Am J Pathol 174, $1017-1026$.

55. Llaverias G, Danilo C, Wang Y, et al. (2010) A Western-type diet accelerates tumor progression in an autochthonous mouse model of prostate cancer. Am J Pathol 177, 3180-3191.

56. Krycer JR, Kristiana I \& Brown AJ (2009) Cholesterol homeostasis in two commonly used human prostate cancer cell-lines, LNCaP and PC-3. PLOS ONE 4, e8496.

57. Krauss RM (2010) Lipoprotein subfractions and cardiovascular disease risk. Curr Opin Lipidol 21, 305-311.

58. Kok DE, van Roermund JG, Aben KK, et al. (2011) Blood lipid levels and prostate cancer risk; a cohort study. Prostate Cancer Prostatic Dis 14, 340-345.

59. Ohlsson C, Mohan S, Sjogren K, et al. (2009) The role of liver-derived insulin-like growth factor-I. Endocr Rev 30, 494-535.

60. Brasky TM, Till C, White E, et al. (2011) Serum phospholipid fatty acids and prostate cancer risk: results from the prostate cancer prevention trial. Am J Epidemiol 173, 1429-1439.

61. Risbridger GP, Davis ID, Birrell SN, et al. (2010) Breast and prostate cancer: more similar than different. Nat Rev Cancer 10, 205-212.

62. Hoffmann I (2003) Transcending reductionism in nutrition research. Am J Clin Nutr 78, 514S-516S. 